\section{(A) Check for updates}

Cite this: Nanoscale, 2020, 12 , 22340

DOI: $10.1039 /$ dOnr90231d

rsc.li/nanoscale

\title{
Correction: Recent development of two- dimensional metal-organic framework derived electrocatalysts for hydrogen and oxygen electrocatalysis
}

\author{
Hengbo Wu, ${ }^{a}$ Jie Wang, ${ }^{c}$ Wei Jin*a and Zexing Wu*b
}

Correction for 'Recent development of two-dimensional metal-organic framework derived electrocatalysts for hydrogen and oxygen electrocatalysis' by Hengbo Wu et al., Nanoscale, 2020, 12, 18497-18522 DOI: 10.1039/DONR04458J.

The authors regret that statements acknowledging reproduced work were omitted from a number of the captions of figures (Fig. 1-18) in the original manuscript, including the graphical abstract. The figures containing reproduced work, along with updated captions containing the acknowledgement statements, are displayed in this notice. The references in the captions are as they appear in the original manuscript. In addition, a description of Fig. $18 \mathrm{f}-\mathrm{h}$ was mistakenly omitted from section 4.2.2, 'OER and ORR'. These descriptions are as displayed immediately below:

The discharge and charge polarization curves displayed a higher peak power density over Pt/C-IrO 2 (Fig. $18 \mathrm{f}$ and g). Fig. $18 \mathrm{~h}$ shows significant battery performance by using an optimized three electrode configuration.

Figures

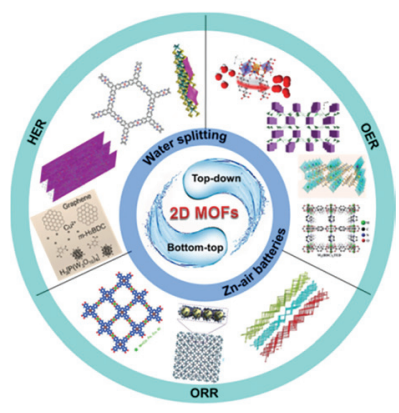

Reprinted with permission from ref. 49. Copyright (2019) American Chemical Society. Reprinted with permission from ref. 137. Copyright (2017) American Chemical Society. Reproduced with permission from ref. 145. Copyright (2015) Wiley-VCH. Reproduced with permission from ref. 159. Copyright (2019) Wiley-VCH. Reproduced with permission from ref. 172. Copyright (2020) Royal Society of Chemistry. Reproduced with permission from ref. 171. Copyright (2019) Wiley-VCH. Reproduced with permission from ref. 170. Copyright (2019) Royal Society of Chemistry. Reproduced with permission from ref. 169. Copyright (2019) Royal Society of Chemistry. Reproduced with permission from ref. 130. Copyright (2019) Wiley-VCH. Reproduced with permission from ref. 63. Copyright (2017) Royal Society of Chemistry. Reproduced with permission from ref. 204. Copyright (2019) Wiley-VCH.

\footnotetext{
${ }^{a}$ School of Chemical and Material Engineering, Jiangnan University, Wuxi 214122, China. E-mail: wjin@ipe.ac.cn

${ }^{b}$ College of Chemistry and Molecular Engineering, Qingdao University of Science \& Technology, Qingdao 266042, PR China. E-mail: splswzx@qust.edu.cn

${ }^{c}$ College of Chemistry and Pharmaceutical Sciences, Qingdao Agricultural University, Qingdao, 266109, China
} 


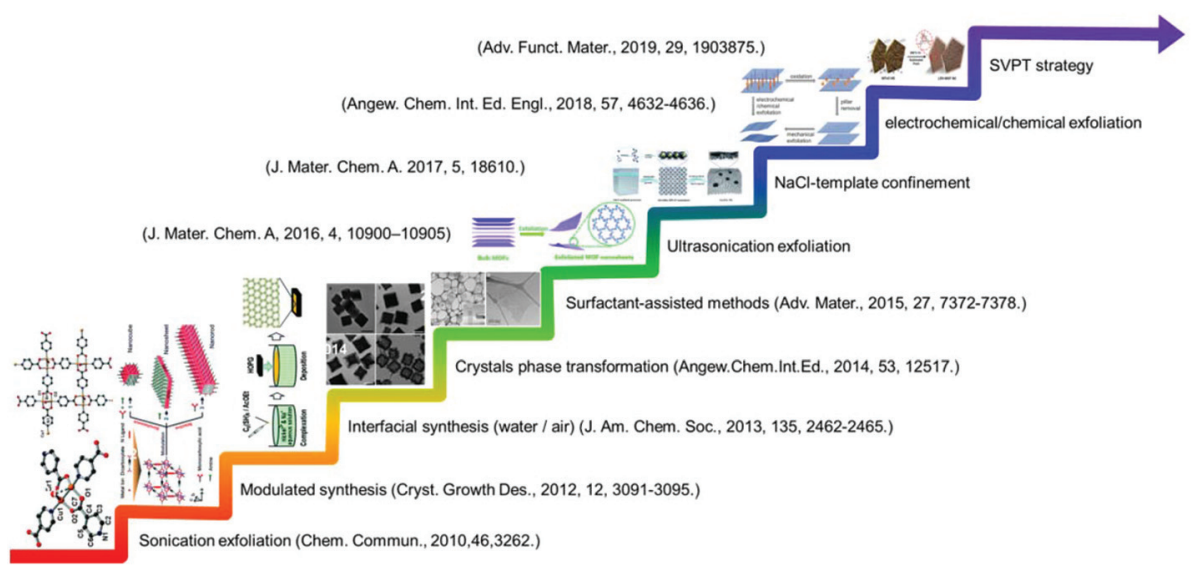

Fig. 1 Timeline of important breakthroughs in the synthesis of 2D MOF nanosheets. Reproduced with permission from ref. 57. Copyright (2010) Royal Society of Chemistry. Reprinted with permission from ref. 58. Copyright (2012) American Chemical Society. Reprinted with permission from ref. 59. Copyright (2013) American Chemical Society. Reproduced with permission from ref. 60. Copyright (2014) Wiley-VCH. Reproduced with permission from ref. 61. Copyright (2015) Wiley-VCH. Reproduced with permission from ref. 62. Copyright (2016) Royal Society of Chemistry. Reproduced with permission from ref. 63. Copyright (2017) Royal Society of Chemistry. Reproduced with permission from ref. 64. Copyright (2018) Wiley-VCH. Reproduced with permission from ref. 65. Copyright (2019) Wiley-VCH.

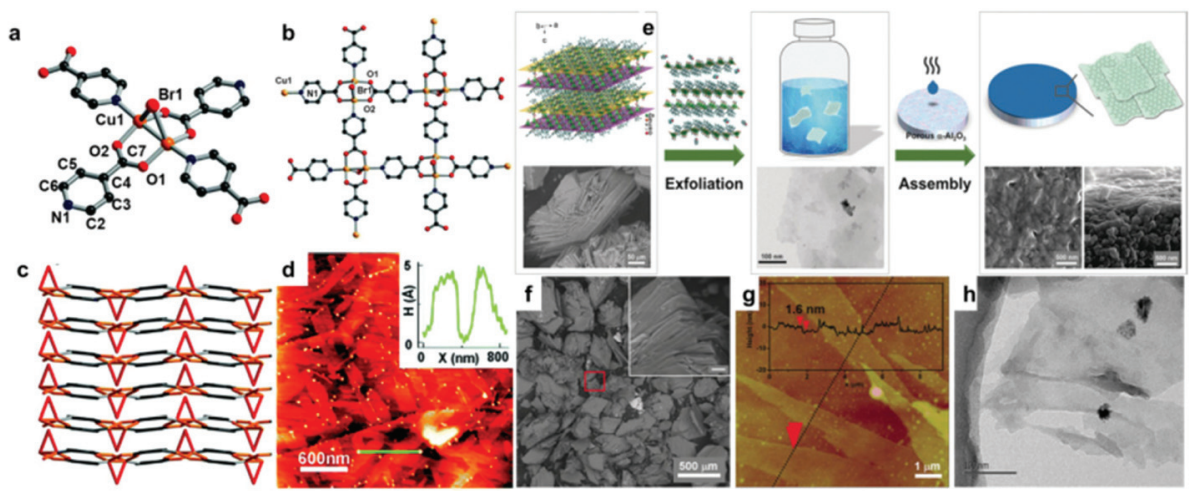

Fig. 2 (a) Detailed environment of the $\mathrm{Cu}$ coordinated in $\left[\mathrm{Cu}_{2} \mathrm{Br}(\mathrm{IN})_{2}\right]_{n}$ crystals. The stacking of $\left[\mathrm{Cu}_{2} \mathrm{Br}(\mathrm{IN})_{2}\right]_{n}$ in different axes, top view (b), side view (c). (d) AFM image and height profile of $\left[\mathrm{Cu}_{2} \mathrm{Br}\left(\mathrm{IN}_{2}\right]_{n}\right.$ nanosheets. Reproduced with permission from ref. 57 . Copyright 2010 Royal Society of Chemistry. (e) Schematic illustration of the synthesis procedure towards $Z n_{2}(\text { bim })_{3}$ nanosheets. (f) SEM image of the as-synthesized $Z n_{2}(\text { bim) })_{3}$ precursor. The inset image shows an enlarged area marked by the red frame (the scale: $10 \mu \mathrm{m})$. AFM image (g) and TEM image (h) of exfoliated $\mathrm{Zn}_{2}(\text { bim })_{3}$ nanosheets. Reproduced with permission from ref. 76. Copyright (2017) Wiley-VCH. 


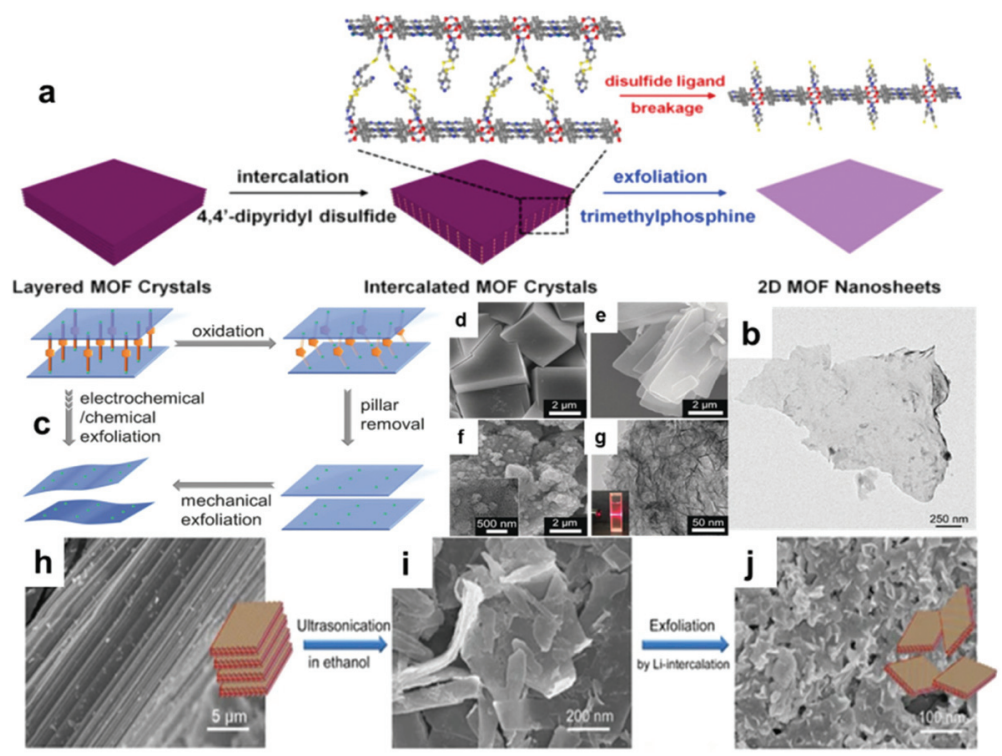

Fig. 3 (a) Representation of the synthesis of 2D MOF nanosheets via an intercalation and chemical exfoliation approach. (b) TEM image of a single layer exfoliated MOF nanosheet. Reprinted with permission from ref. 78. Copyright (2017) American Chemical Society. (c) Selective pillar removal and the exfoliation process of the pillared-layer MOF into dispersed nanosheets. SEM images of (d) 3D-Co, (e) 2D-Co, and (f) 2D-Co-NS. (g) TEM image of 2D-Co-NS. Reproduced with permission from ref. 64. Copyright (2018) Wiley-VCH. (h-j) Schematic illustration of the Li-intercalation synthesis for MOF-Ln. Reproduced with permission from ref. 79. Copyright (2017) Springer Nature.
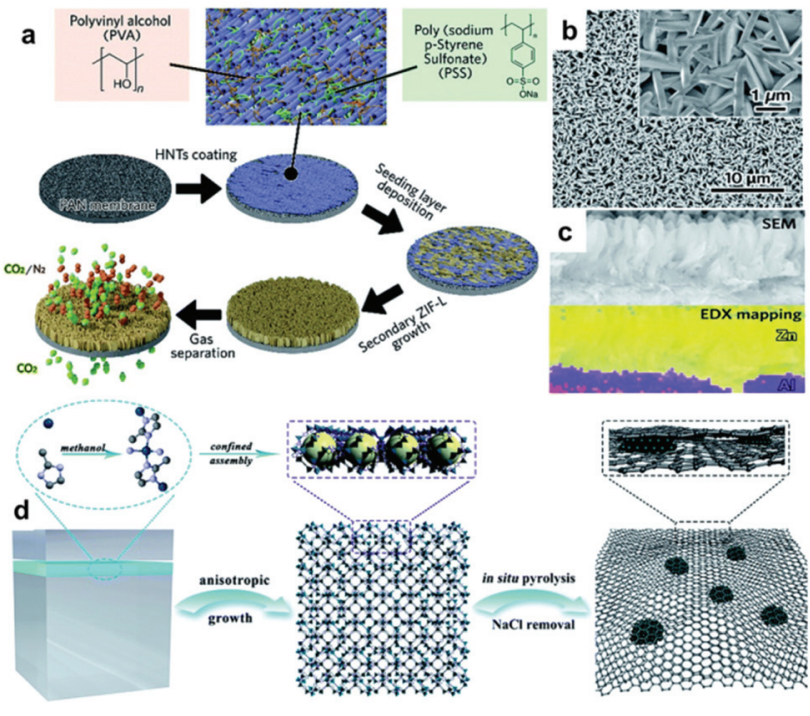

$\mathrm{NaCl}$ confined precursor
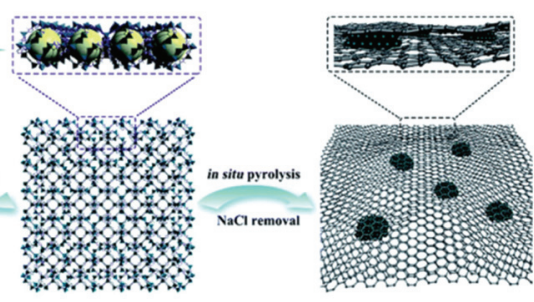

ultrathin ZIF-67 nanosheets

Co,N-C NS

Fig. 4 (a) Schematic diagram of the PAN-HNTs-ZIF-L membrane growth processes. (b) SEM image of the top-surface of the PAN-HNTs-ZIF-L membrane. (c) EDX mapping and reference SEM image of the PAN-HNTs-ZIF-L membrane cross-sectional region. Reproduced with permission from ref. 85. Copyright (2019) Royal Society of Chemistry. (d) Schematic illustration of the synthesis of ZIF- 67 nanosheets through the salt-template method. Reproduced with permission from ref. 63. Copyright (2017) Royal Society of Chemistry. 

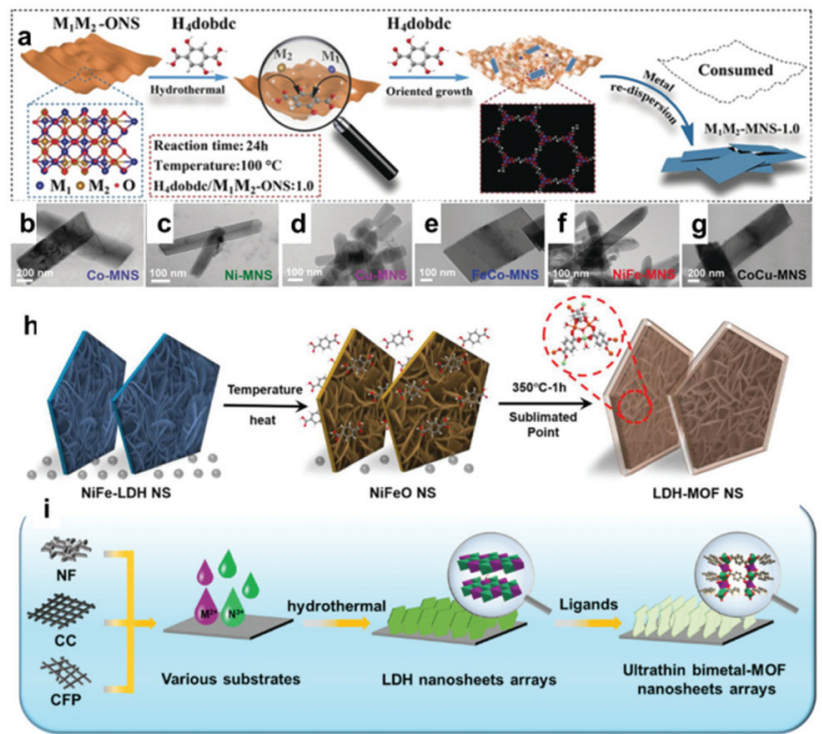

Fig. 5 (a) Schematic illustration of transforming metal oxide nanosheets to MOF nanosheets via the 2dOSA strategy. (b-g) TEM images of Co-MNS, Ni-MNS, Cu-MNS, FeCo-MNS, NiFe-MNS, and CoCu-MNS. Reproduced with permission from ref. 87. Copyright (2019) Wiley-VCH. (h) Synthesizing LDH-MOF NS using a SVPT strategy. Reproduced with permission from ref. 65. Copyright (2019) Wiley-VCH. (i) Schematic illustration of the synthetic route to ultrathin bimetal-MOF nanosheet arrays on the 3D conductive matrix. Reproduced with permission from ref. 88. Copyright (2019) Wiley-VCH.
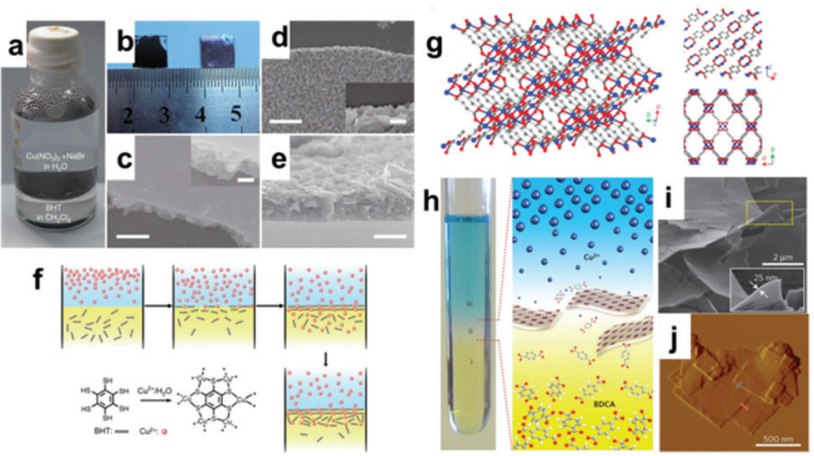

Fig. 6 (a) Photograph of the Cu-BHT MOF forming at the $\mathrm{H}_{2} \mathrm{O} / \mathrm{CH}_{2} \mathrm{Cl}_{2}$ interface. (b) Photograph of upside-up (right) and upside-down (left) films transferred on glass substrates. The SEM image of (c) top side, (d) down side and (e) cross-section surface of the as-synthesized Cu-BHT MOF. ( $f$ ) Scheme of the formation of Cu-BHT. Reproduced with permission from ref. 89. Copyright (2015) Springer Nature. (g) Crystal structure of 2D CuBDC MOF. (h) Spatial arrangement of the different liquid layers to produce the Cu-BDC nanosheets. SEM (i) and AFM (j) images of Cu-BDC. Reprinted by permission from ref. 95. Copyright (2015) Springer Nature.

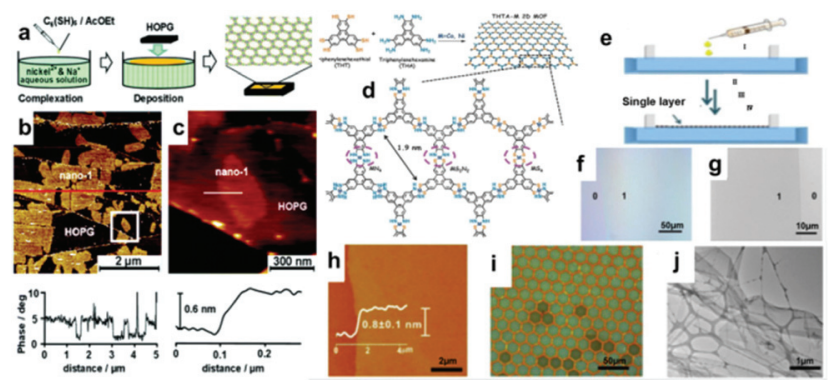

Fig. 7 (a) Schematic illustration of the fabrication process of nano-1 at the water/air interface. (b) AFM phase images of Ni-BHT nanosheets (b) and a single-layer Ni-BHT nanosheet (c) on HOPG. Reprinted with permission from ref. 59. Copyright (2013) American Chemical Society. (d) Schematic synthesis of single-layer THTA-Co. (e) Schematic illustration showing the fabrication of single layer nanosheets using the LB strategy. ( $\mathrm{f}$ and g) OM and SEM images revealing large-area single-layer sheets, respectively. The layer number is shown in the images. (h) AFM image and the corresponding height profile analysis. (i) OM image of single-layer sheets after horizontal transfer onto a copper grid with hexagonal holes (side length = $18 \mu \mathrm{m})$. (j) TEM image of a single layer on a lacey carbon grid. Reproduced with permission from ref. 97. Copyright (2015) Wiley-VCH. 


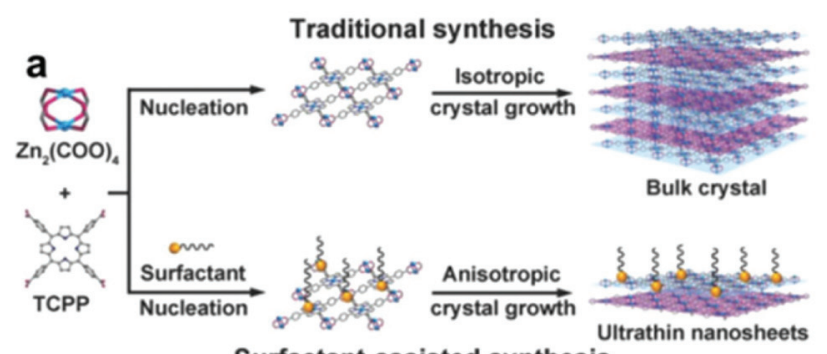

Surfactant-assisted synthesis
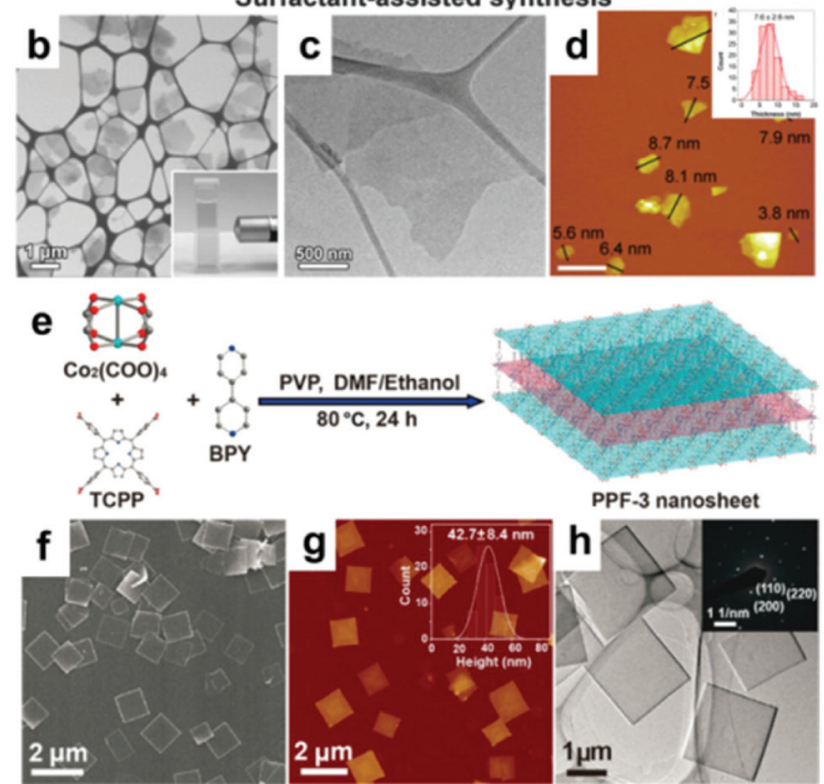

Fig. 8 (a) Conventional synthesis and surfactant-assisted synthesis of the Zn-TCPP MOF. (b) TEM image of the obtained Zn-TCPP nanosheets. (c) TEM image of a single Zn-TCPP nanosheet. (d) AFM image of the Zn-TCPP nanosheets. Inset: Statistical analysis of the thickness of Zn-TCPP nanosheets. Reproduced with permission from ref. 61. Copyright (2015) Wiley-VCH. (e) Schematic illustration of the fabrication procedure for PPF-3 nanosheets. (f) SEM image of PPF-3 nanosheets. (g) AFM image of PPF-3 nanosheets. Inset: Height profile. (h) TEM image of PPF-3 nanosheets. Inset: SAED pattern. Reprinted with permission from ref. 112. Copyright (2016) American Chemical Society.

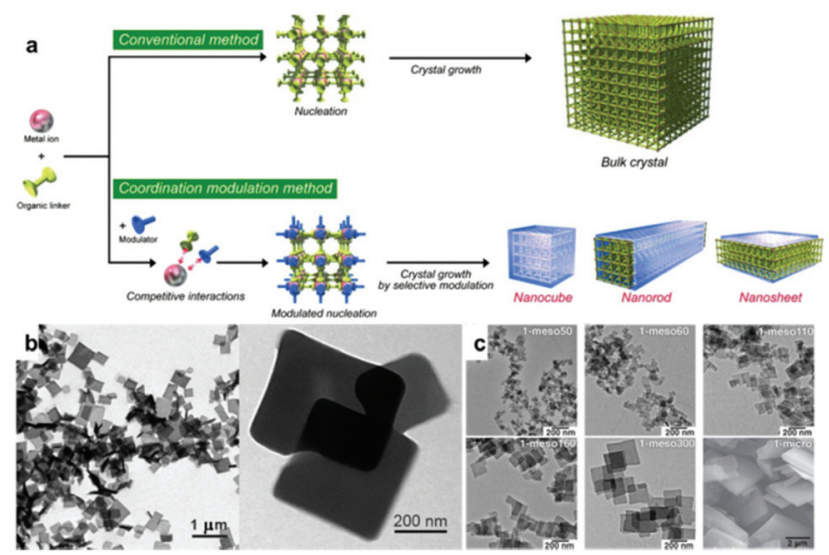

Fig. 9 (a) Schematic illustration of the comparison between the traditional approach and coordination modulation approach. Reproduced with permission from ref. 122. Copyright (2009) Wiley- $\mathrm{VCH}$. (b) TEM images of $2 \mathrm{D}\left[\mathrm{Cu}_{2}(\mathrm{ndc})_{2}(\text { dabco })\right]_{n}$ nanosheets. Reprinted with permission from ref. 58. Copyright (2012) American Chemical Society. (c) TEM images of 1-meso50, 60, 110, 160, 300 and 1-micro. From ref. 123. Reprinted with permission from American Association for the Advancement of Science. 


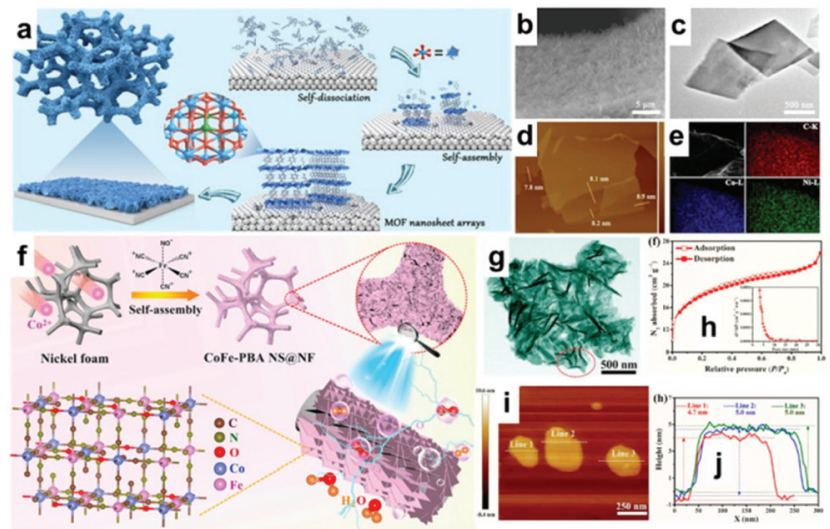

Fig. 10 (a) Schematic illustration for the synthesis of CoNi-MOFNA, (b) SEM image, (c) TEM image, (d) AFM image and (e) EDX elemental mapping images of CoNi-MOFNA. Reprinted from ref. 131. Copyright 2020, with permission from Elsevier. (f) Schematic illustration of the synthesis of the CoFe-PBA NS@NF composite. (h) $\mathrm{N}_{2}$ ad-/desorption isotherms. (g) TEM image, (i) AFM image of the CoFe-PBA nanosheets and its corresponding line-scan profile (j). Reprinted from ref. 128. Copyright 2020, with permission from Elsevier.
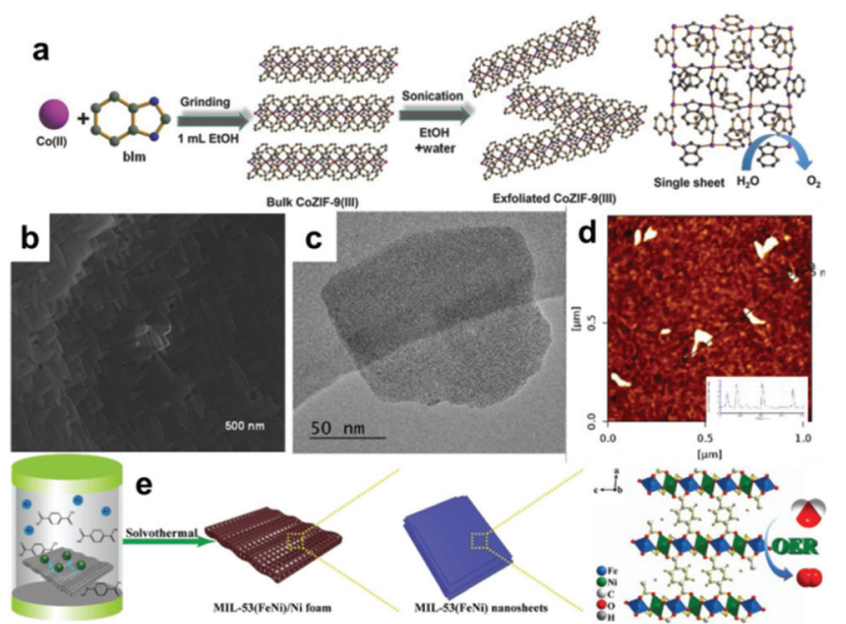

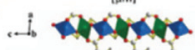
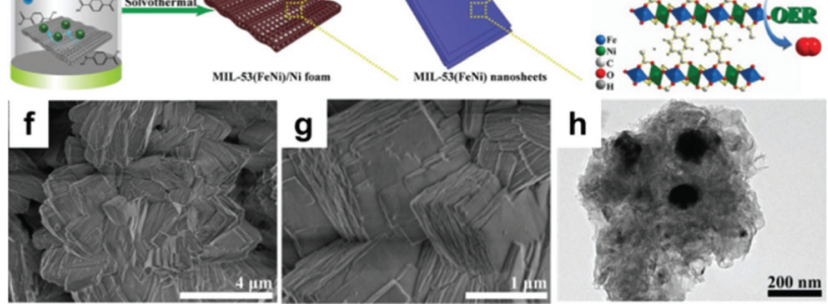

Fig. 11 (a) Schematic presentation of 2D Co-ZIF-9(III) nanosheets exfoliated in the liquid phase from bulk Co-ZIF-9(III). (b) FE-SEM images of bulk Co-ZIF-9. HAADF-TEM (c) and AFM (d) images of liquid exfoliated 2D Co-ZIF-9. Reproduced with permission from ref. 129. Copyright (2018) Wiley$\mathrm{VCH}$. (e) Schematic illustration of the fabrication procedure of MIL-53(FeNi)/NF. ( $f$ and g) SEM images and (h) TEM image of MIL-53(FeNi)/NF. Reproduced with permission from ref. 86. Copyright (2018) Wiley-VCH. 


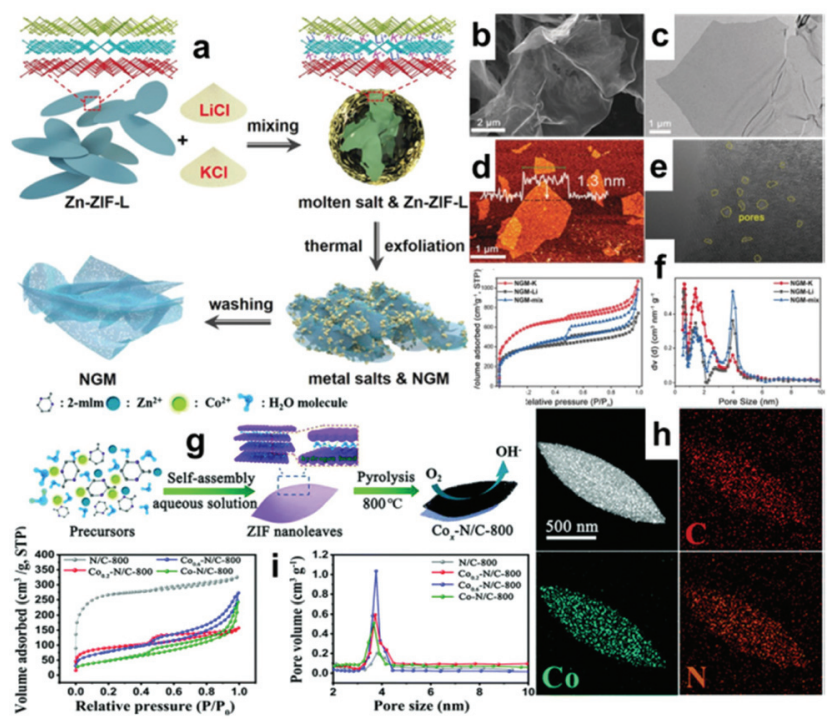

Fig. 12 (a) Illustration of the synthesis method to produce NGM. (b) SEM image, (c) TEM image, (d) AFM image, and (e) HRTEM image of NGM-800 (yellow circles: nanopore). (f) $\mathrm{N}_{2}$ adsorption-desorption isotherms of NGM-800 and the corresponding pore size distribution. Reproduced with permission from ref. 130. Copyright (2019) Wiley-VCH. (g) Schematic illustration of the synthesis process for Co-N/C. (h) EDX-mapping of Co ${ }_{0.6}^{-}$ $\mathrm{N} / \mathrm{C}-800$. (i) $\mathrm{N}_{2}$ adsorption-desorption isotherms and the pore size distribution plots of Co-N/C-800. Reproduced with permission from ref. 135 . Copyright (2018) Royal Society of Chemistry.

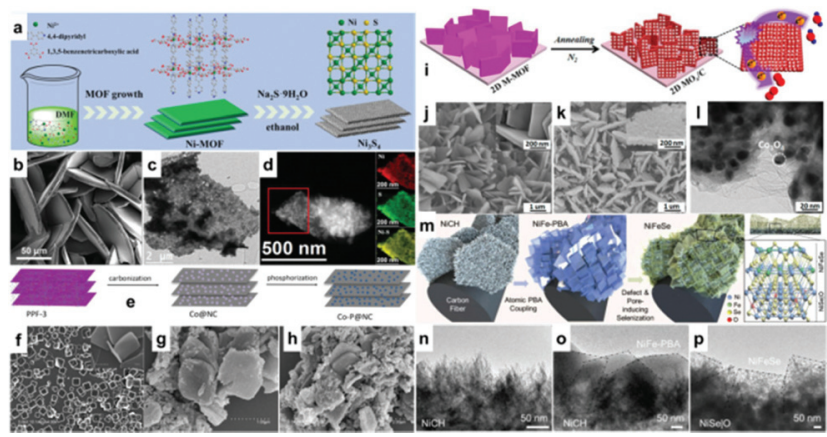

Fig. 13 (a) Schematic illustration of the synthesis of NM50-Ni $\mathrm{S}_{4}$. (b) SEM images of NM50. (c) TEM images of NM50-Ni $\mathrm{S}_{4}$. (d) EDX-mapping of $\mathrm{NM} 50-\mathrm{Ni}_{3} \mathrm{~S}_{4}$ obtained from the area highlighted in red in the STEM image. Reproduced with permission from ref. 134. Copyright (2019) Wiley-VCH. (e) Schematic fabrication for the synthesis of Co-PaNC. SEM images of (f) PPF-3 nanosheets, (g) Co@NC-800, and (h) Co-P@NC-800. Reprinted with permission from ref. 137. Copyright (2017) American Chemical Society. (i) Schematic representation of fabricating $\mathrm{MO}_{x} / \mathrm{C}$ arrays. (j) SEM image of 2D Co-BDC; SEM (k) and TEM (l) images of $2 \mathrm{D} \mathrm{CO} \mathrm{CO}_{3} / \mathrm{C}_{\mathrm{BDC}}$ arrays. Reprinted with permission from ref. 136. Copyright (2018) American Chemical Society. $(\mathrm{m})$ Schematic route for the synthesis of NiFeSeaNiSelO@CC. TEM images of (n) NiCH@CC, (o) NiFe-PBA@NiCHaCC, and (p) NiFeSeaNiSel OaCC. Reproduced with permission from ref. 144. Copyright (2019) Wiley-VCH. 
a

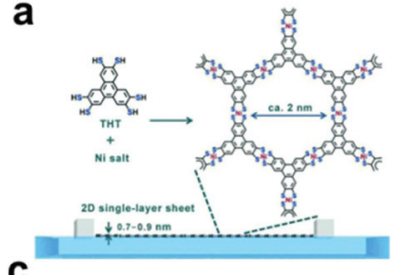

C

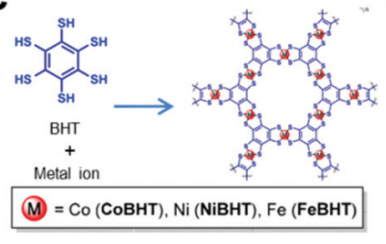

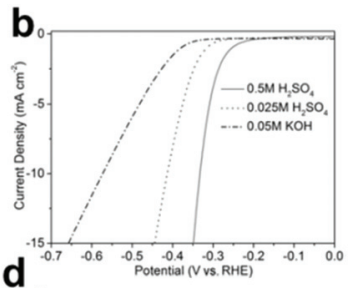

d

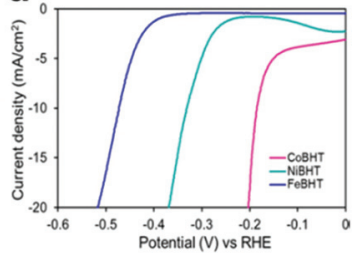

Fig. 14 (a) Schematic illustration of the synthesis process of 2D SP single-layer sheets. (b) HER polarization diagrams in different electrolyte solutions. Reproduced with permission from ref. 145. Copyright (2015) Wiley-VCH. (c) Schematic illustration of the synthesis of 2D MOFs (Co-BHT, Ni$\mathrm{BHT}$, and Fe-BHT). (d) LSV curves. Reprinted with permission from ref. 147. Copyright (2018) American Chemical Society.

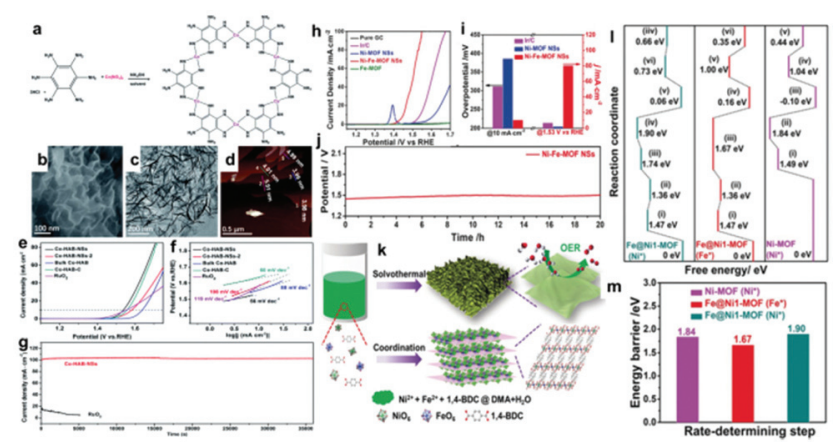

Fig. 15 (a) Synthetic scheme of Co-HAB-NSs. SEM image (b), TEM image (c) and (d) AFM image of Co-HAB-NSs. (e) LSV curves of Co-HAB-NSs, $\mathrm{Co}-\mathrm{HAB}-\mathrm{NSs}-2$, bulk $\mathrm{Co}-\mathrm{HAB}, \mathrm{Co}-\mathrm{HAB}-\mathrm{C}$ and $\mathrm{RuO}_{2}$. (f) The corresponding Tafel plots. (g) $1-t$ curves of Co-HAB-NSs and RuO ${ }_{2}$ at 1.75 V. Reproduced with permission from ref. 196. Copyright (2020) Royal Society of Chemistry. (h) LSV curves of pure GC, commercial Ir/C, Ni-MOF NSs, Fe-MOF, and Ni-Fe-MOF NSs. (i) The corresponding overpotentials and current densities of different catalysts at $10 \mathrm{~mA} \mathrm{~cm}^{-2}$ and $1.53 \mathrm{~V}$. (j) Chronopotentiometry curves of the Ni-Fe-MOF NSs at $10 \mathrm{~mA} \mathrm{~cm}^{-2}$. (k) Synthetic procedure of ultrathin Ni-Fe-MOF nanosheets. (l) Standard free energy diagram of Ni-MOF $\left(\mathrm{Ni}^{*}\right)$, FeaNi1-MOF $\left(\mathrm{Fe}^{*}\right)$, and Fe@Ni1-MOF $\left(\mathrm{Ni}^{*}\right)$ and the corresponding energy barrier of the rate-determining step $(\mathrm{m})$. Reproduced with permission from ref. 198. Copyright (2019) Wiley-VCH.

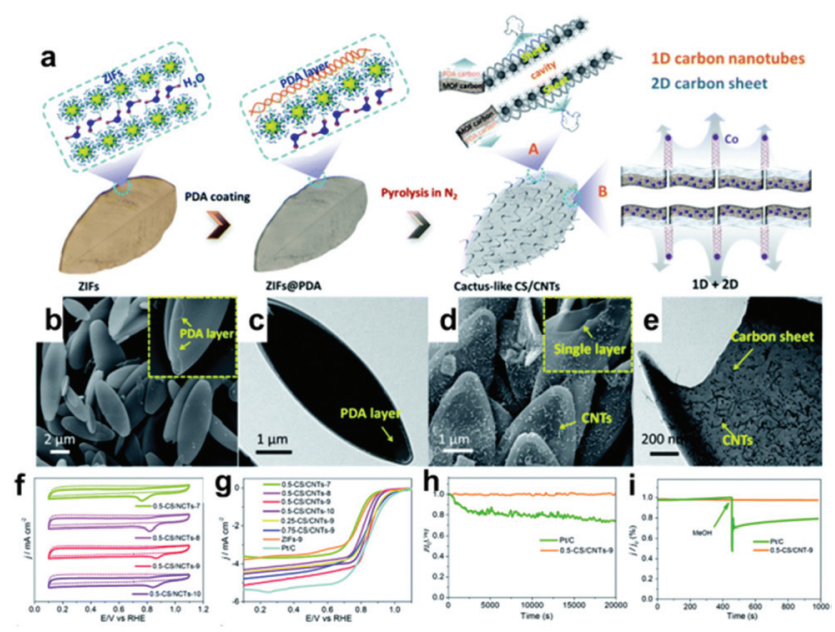

Fig. 16 (a) Schematic illustration of the synthetic route of CS/CNTs; the SEM (b) and TEM (c) images of ZIFs@PDA, respectively. The SEM (d) and TEM (e) images of 0.5-CS/CNTs-900; (f) the CV curves of 0.5-CS/CNTs-7/8/9/10; (g) the LSV curves of Pt/C, ZIFs-9, 0.25/0.75-CS/CNTs-9 and 0.5$\mathrm{CS} / \mathrm{CNTs}-7 / 8 / 9 / 10$; (h) the long-term durability and (i) methanol tolerance of 0.5 -CS/CNTs-9 in $\mathrm{O}_{2}$-saturated $0.1 \mathrm{M} \mathrm{KOH}$ solution. Reproduced with permission from ref. 201. Copyright (2019) Royal Society of Chemistry. 


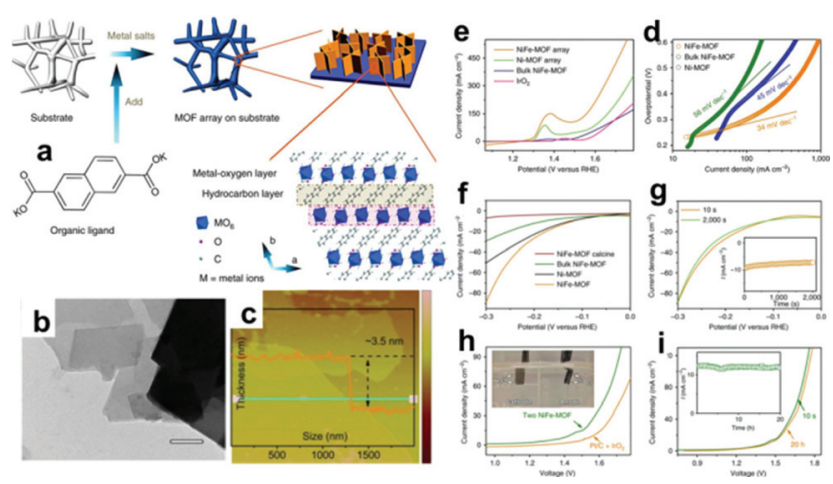

Fig. 17 (a) Schematic illustration of the formation of Ni/Fe-MOF nanosheets. (b) TEM image. (c) AFM image and the corresponding height profile along. (d) LSV plots obtained with NiFe-MOF, Ni-MOF, bulk NiFe-MOF and $\operatorname{IrO}_{2}$ for OER at $10 \mathrm{mV} \mathrm{s}^{-1}$ in $0.1 \mathrm{M}$ KOH. (e) Tafel plots. (f) LSV plots obtained with NiFe-MOF, bulk NiFe-MOF, Ni-MOF and calcined NiFe-MOF for HER at $10 \mathrm{mV} \mathrm{s}^{-1}$ in $0.1 \mathrm{M}$ KOH. (g) Polarization curves before and after an accelerated degradation test for HER; inset: the corresponding chronoamperometric profile. (h) Water electrolysis polarization curves for NiFe-MOF in a two-electrode configuration at $10 \mathrm{mV} \mathrm{s}^{-1}$ in $0.1 \mathrm{M} \mathrm{KOH}$; inset: a photograph of hydrogen and oxygen gas bubbles at the NiFe-MOF electrodes at an applied cell voltage of $1.6 \mathrm{~V}$. (i) LSV plots before and after $20 \mathrm{~h}$ chronoamperometric tests at a cell voltage of $1.5 \mathrm{~V}$ for water splitting; inset: the corresponding chronoamperometric plot. Reproduced with permission from ref. 209. Copyright (2017) Springer Nature.

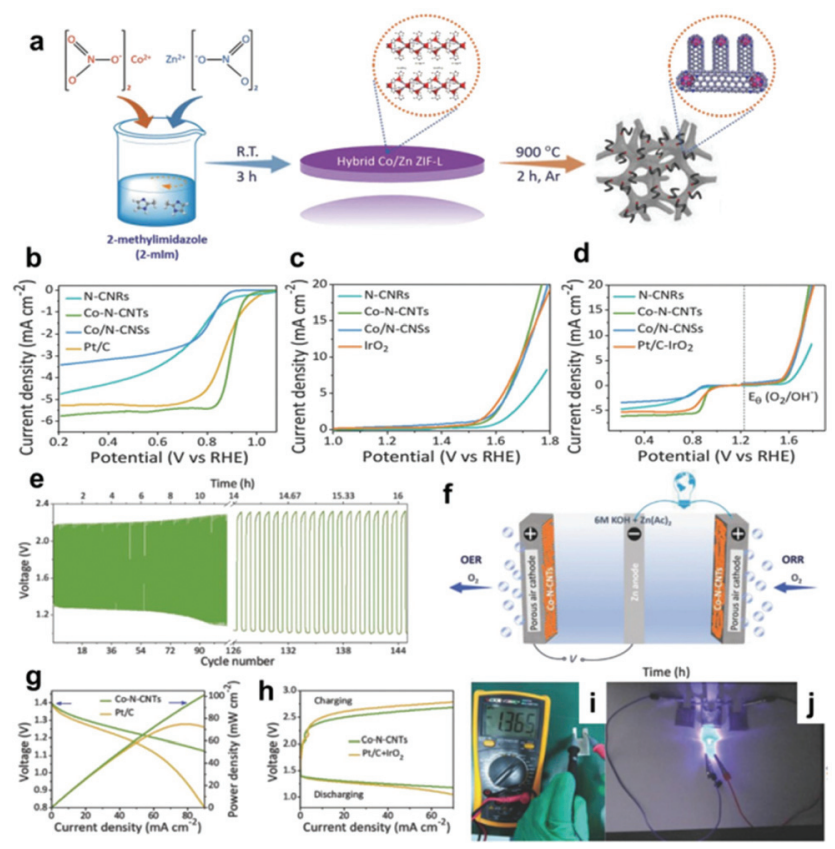

Fig. 18 (a) Representation of the preparation of 2D Co/Zn ZIF-L and Co-N-CNTs. LSV curves of different catalysts for ORR (b) and OER (c) in $\mathrm{O}_{2}-$ saturated $0.1 \mathrm{M} \mathrm{KOH}$ at $1600 \mathrm{rpm}$. (d) The overpotentials between the $E_{1 / 2}$ of ORR and $E_{\mathrm{j}_{10}}$ of the OER. (e) Long-term cycling performance at $2 \mathrm{~mA}$ $\mathrm{cm}^{-2}$ with the Co-N-CNT catalyst as the air-cathodes. (f) Schematic of the $\mathrm{Zn}$-air battery. (g) Discharge polarization curves and the corresponding power density of zinc-air batteries. (h) Charge and discharge polarization curves. (i) Photograph of the assembled all-solid-state $\mathrm{Zn}$-air battery measured with a voltammeter. (j) Photograph of a lighted green LED. Reproduced with permission from ref. 236. Copyright (2017) Wiley-VCH.

The Royal Society of Chemistry apologises for these errors and any consequent inconvenience to authors and readers. 